\title{
Archéopages
}

Archéopages

Archéologie et société

34 | 07/2012

Campagnes

\section{Un point sur la photogrammétrie}

Caroline Delevoie, Bruno Dutailly, Pascal Mora et Robert Vergnieux

\section{(2) OpenEdition}

\section{Journals}

Édition électronique

URL : https://journals.openedition.org/archeopages/410

DOI : 10.4000/archeopages.410

ISSN : 2269-9872

\section{Éditeur}

INRAP - Institut national de recherches archéologiques préventives

\section{Édition imprimée}

Date de publication : 1 février 2012

Pagination : 86-89

ISSN : $1622-8545$

\section{Référence électronique}

Caroline Delevoie, Bruno Dutailly, Pascal Mora et Robert Vergnieux, « Un point sur la

photogrammétrie », Archéopages [En ligne], 34 | 07/2012, mis en ligne le 01 juillet 2012, consulté le 21 janvier 2022. URL : http://journals.openedition.org/archeopages/410 ; DOI : https://doi.org/10.4000/ archeopages.410 


\section{Du bon usage de la 3D en archéologie}

En archéologie, la vue $3 \mathrm{D}$ s'avère très utile à la conception d'illustrations pédagogiques et esthétiques destinées à la valorisation des sites auprès du grand public. Cette méthode peut également être conçue comme un outil essentiel à l'approfondissement de la réflexion des chercheurs.

Dans le domaine de la gestion du patrimoine architectural et archéologique, les techniques de relevé telles que la photogrammétrie et l'ortho-photographie sont utilisées de longue date dans la constitution des fonds documentaires et graphiques : le plan, la coupe et l'élévation à une échelle définie constituent la base de toute

\section{Un point sur la photogrammétrie}

Caroline Delevoie, CNRS, UPS 3551 «Archéovision » Bruno Dutailly, CNRS, UPS 3551 «Archéovision » et UMR 5199 «PACEA»

Pascal Mora, Archéotransfert, Cellule de transfert de l'UPS 3551 "Archéovision »

Robert Vergnieux, CNRS, UPS 3551 "Archéovision »

\section{La numérisation ${ }_{3} D$ sur le terrain}

De nombreux cas d'application de la numérisation 3D (complète ou partielle) de sites archéologiques ont permis de démontrer l'intérêt de cette approche par rapport aux techniques traditionnelles de relevé, en terme de rapidité, de quantité et de fiabilité des informations collectées, des possibilités de traitement post-fouille, des possibilités de valorisation, etc. Cependant, jusqu'à très récemment, l'utilisation de la numérisation ${ }_{3} \mathrm{D}$ de terrain restait anecdotique à cause principalement de son coût élevé induit par l'utilisation de matériel lourd type scanners $3 \mathrm{D}$ dont les coûts d'acquisition ou de prestation sont très élevés. Issus du monde industriel, le type de matériel utilisé, bien que très performant et pointu, exige du personnel qualifié pour sa manipulation et génère souvent d'énormes quantités de données dont la gestion et les traitements impliquent au mieux des coûts supplémentaires non prévus initialement et au pire une sous-utilisation voire forme d'étude sur l'objet en question. Plus récemment, le développement de systèmes de relevé automatisés ont permis la modélisation par informatique de ces mêmes objets en trois dimensions, offrant de nouvelles perspectives pour l'enregistrement des vestiges. La modélisation $3 \mathrm{D}$ des vestiges archéologiques apparait, dans la chaine graphique et les relevés des opérations archéologiques, comme un triple progrès : son acquisition est simple et rapide au moyen de la photographie ; elle dépasse les contraintes de temps et d'espace; enfin, elle ouvre des perspectives scientifiques et comporte un fort potentiel de communication.

un abandon de ces données. Depuis quelques années, la situation est en train d'évoluer : on assiste à l'émergence de nouvelles méthodes de numérisation $3 \mathrm{D}$ entièrement informatiques désignées sous le terme de photogrammétrie par corrélation dense ou bien encore par corrélation d'images. Cependant, l'acquisition par scanner laser ou longue portée est encore très adaptée à certaines situations. En effet, la photogrammétrie est peu efficace en condition d'éclairage non uniforme, et sur des surfaces brillantes ou des aplats de couleurs uniformes. C'est le cas en grotte, par exemple, où il est difficile d'avoir un éclairage constant sur de grandes distances. Il faudrait avoir un grand nombre de flashs fixes, ou un flash annulaire très puissant, ou des spots alimentés en courant électrique (batteries puissantes ou alimentation secteur). Un autre exemple concerne les milieux très humides où les surfaces recouvertes de condensation brillent. Si les besoins des relevés à effectuer concernent essentiellement la géométrie d'un site sans accorder une grande importance à la texture, les solutions utilisant des scanners laser ou longue portée permettent une acquisition rapide et efficace, et viennent en complément des techniques par photogrammétrie. La conjugaison des différentes techniques amène à constituer un relevé complet de site avec des résolutions 
de numérisation et des qualités de texture adaptées aux besoins. Il est donc important de définir en amont et dans sa globalité quels sont les besoins des équipes scientifiques pour éviter la course à la numérisation la plus fine possible engendrant des coûts démesurés et des modèles $3 \mathrm{D}$ impossibles à manipuler.

Nous voulons ici évoquer plus précisément les principes de la photogrammétrie dont l'usage semble avoir un avenir fondamental pour l'archéologie de demain. L'emploi du terme photogrammétrie peut cependant prêter à confusion, car cette technique est apparue, sous ses formes les plus rudimentaires, très peu de temps après la photographie au milieu du XIX ${ }^{\mathrm{e}}$ siècle et elle n'a d'ailleurs pas cessée d'évoluer depuis. La photogrammétrie par corrélation d'image, bien que basée sur des principes de base similaires, constitue une des dernières évolutions de cette technique et donne des résultats sans commune mesure avec ceux obtenus il y a seulement quelques décennies.

\section{Principe général de la photogrammétrie} Il consiste à retrouver la position dans l'espace de chaque point de la surface visible d'un sujet photographié à partir de l'analyse de plusieurs photographies prises sous plusieurs angles de vue différents. Une photographie est une projection plane (sur le capteur de l'appareil photo) de l'image d'un objet tridimensionnel, le processus photogrammétrique effectue en quelque sorte l'opération inverse, il retrouve la position des points dans l'espace à partir de l'analyse des images. Les calculs mis en jeu sont complexes et nombreux, détection et appariement des points homologues sur les photographies, triangulation, autocalibration, calcul épipolaire etc. le résultat final se traduit par un nuage de points d'une densité variable en fonction des paramètres choisis et de la qualité des photographies utilisées. Dans la pratique, les phases de ce procédé sont effectuées par des programmes distincts qui fonctionnent successivement et s'enchainent de manière automatique. Même s'il existe plusieurs solutions logicielles différentes, on retrouve très souvent la même organisation, avec quatre principales étapes distinctes : détection de détails identiques sur différentes images (les clés), cette identification doit être robuste et peu sensible à l'orientation ou au changement d'échelle (zoom, distance de prise de vue); mise en correspondance des clés des différentes vues - ces deux phases sont réalisées le plus souvent par le programme SIFT et ses dérivés (Lowe, 2004) ; calcul de leurs positions dans l'espace - cette phase permet également de calibrer les images (autocalibration) de manière à retirer toutes les déformations dues aux imperfections des optiques de l'appareil photo, elle peut être réalisée avec le logiciel Bundler (Snavely, 2006, 2007); enfin, une phase de reconstruction dense qui utilise ces données de calibration pour calculer un nuage de points bien plus dense que celui constitué par les seules clés du début - cette tâche peut être confiée au logiciel PMVS (Furukawa, Ponce, 2009) ou bien encore au programme Micmac développé par l'IGN (Pierrot-Deseilligny, 2006). Dans les deux cas, la densité finale est paramétrable et plusieurs densités sont possibles en fonction des besoins ; plus une image a une définition élevée, plus elle est susceptible de fournir un nuage de points détaillé. Chaque point reçoit la couleur des pixels de l'image qui ont servi à effectuer les opérations de calcul, ainsi aucune manipulation de texturage n'est nécessaire. La plupart des briques logicielles qui constituent cette chaine sont directement issues des laboratoires de recherche en informatique et ont été mise en ligne pour une utilisation libre. Ceci, combiné à la constante montée en puissance des appareils photo numériques, des ordinateurs et des algorithmes, a permis l'engouement pour ces techniques que l'on observe actuellement auprès du public, mais aussi des professionnels de la numérisation $3 \mathrm{D}$. Car, bien que cette méthode soit totalement logicielle (à part la phase d'acquisition qui exige un appareil photo), les résultats obtenus peuvent être d'excellente qualité et parfaitement comparables à ceux obtenus avec les scanners $3 \mathrm{D}$ de courte ou de longue portée.

\section{Les avantages de la photogrammétrie}

Un des avantages, et non des moindres, qu'apportent ce procédé est le coût relativement faible de la phase d'acquisition, puisqu'il se réduit à l'investissement matériel d'un appareil photo numérique de bonne qualité et au temps de prise de vue. À la condition d'utiliser une méthodologie de prise de vue adaptée, l'archéologue peut effectuer lui-même l'acquisition 3D du sujet qu'il jugera utile de numériser pour sa recherche. Le procédé d'acquisition est donc rapide et ne perturbe pas ou peu la fouille. En ce qui concerne la précision, les publications indiquent une précision de l'ordre de 1 à $0,5 \%$ de la taille de la zone photographiée ; les essais que nous avons menés montrent que l'on peut obtenir des modèles $3 \mathrm{D}$ avec une précision meilleure que le 1 pour 1000 (c'est-à-dire $1 \mathrm{~mm}$ pour $1 \mathrm{~m}$ ou $1 \mathrm{~cm}$ pour $10 \mathrm{~m}$ ) à la condition que les images soient de très bonne qualité [ill. 1]. Le texturage des zones numérisées se fait automatiquement lors du calcul. Comme les sommets du nuage de points sont calculés à partir des pixels des images, ils prennent la couleur du ou des pixels correspondants avec le même niveau de précision. Cette information de couleur peut être conservée lors d'une éventuelle phase de maillage ; la qualité de ce type de colorisation dépend donc de la densité du nuage de points initial. Il est cependant possible d'utiliser les résultats du calcul de la position de l'appareil photo au moment de la prise de vue pour faire une projection des photographies correspondantes sur le maillage avec un positionnement très précis et une définition qui est celle des photographies ayant servi au calcul [ill.2]. 
Du fait de sa mise en œuvre simple, ce procédé est utilisable dans les conditions les plus variées : zone encombrée, milieu subaquatique, peu de recul, impossibilité d'utiliser un trépied lourd... un atout considérable! De plus, il permet l'exploitation de photos aériennes pour la numérisation de sites étendus; des photographies prises à l'aide de ballon captif ou de drone sont parfaitement utilisables.

Alors qu'il faut généralement changer de modèle de scanner laser pour numériser des sujets de taille différente, la photogrammétrie par corrélation d'images permet d'opérer sur des objets de quelques millimètres à plusieurs centaines de mètres, la précision restant toujours relative à la taille de la zone numérisée. Des objets pourront donc également être numérisés, bien que l'acquisition soit plus délicate, car elle nécessite plus d’opérations du fait qu'un objet repose nécessairement sur un support qui masque une partie de sa surface [ill. 3]. Enfin, comme la phase de prise de vue constitue la véritable phase d'acquisition $3 \mathrm{D}$, cela offre à l'archéologue la possibilité de traiter ou non les images. Ainsi, dès lors que les photographies ont été prises correctement, l'archéologue sait qu'il pourra accéder si nécessaire à toutes les informations $3 \mathrm{D}$ potentiellement contenues dans ses images. Ces données doivent pouvoir être pérennisées 1

\section{Les limites du procédé}

Le calcul photogrammétrique utilise les informations contenues dans la carte de pixels que constitue une photographie numérique ; cette information doit donc être la moins dénaturée possible. Pour un résultat optimal, il faut que la qualité des prises de vue soit aussi parfaite que possible. Or, s'il est simple de faire des photographies, il peut s'avérer difficile de faire des photographies remplissant tout les critères de qualité pour le calcul. Les paramètres tels que la profondeur de champ, l'éclairage, le bougé, la qualité de l'optique doivent être maitrisés. La lumière est un facteur très important : son intensité conditionnera plusieurs paramètres de prise de vue; par exemple, un site bien éclairé sera plus facilement photographié, diaphragme et vitesse seront plus confortables et la profondeur de champ n'en sera que plus facilement maîtrisable. Mais un éclairage direct intense provoque des ombres nettes et un grand contraste qui peuvent gêner l'interprétation des images par les algorithmes et dégrader la qualité des textures. Les cas les plus problématiques se rencontrent dans les lieux mal éclairés et qui nécessitent l'emploi de flashs multiples pas toujours faciles à maitriser : grottes, caves, cryptes... Mais de bons résultats ont été obtenus pour peu que ces zones ne soient pas trop volumineuses (impossibilité d'éclairage au flash). Ces points doivent cependant être relativisés en fonction de la résolution et de la précision souhaitées, des tests réalisés avec des smartphones sur des sujets en extérieur ont pu donner des résultats satisfaisants, tout dépend de la résolution et de la précision recherchées.
Les applications possibles

Elles sont identiques à celle des données $3 \mathrm{D}$ denses au sens large.

- soit par le biais de l'utilisation directe du modèle $3 \mathrm{D}$ obtenu, mais cela nécessite déjà une bonne maitrise des outils $3 \mathrm{D}$ classiques d'analyse et de gestion des nuages de points : réalisation de sections et de coupes avec une grande précision (plusieurs milliers de points) ; étude de la géométrie 3D (mesures de volumes, reliefs etc ...).

- soit par le biais de l'utilisation d'ortho-images synthétisées à partir des nuages de points : aide au relevé architectural au sens large ; aide au relevé pierre à pierre (façades, arases de murs, zone de fouille) pour les zones vastes ou difficilement accessibles sans échafaudages ; relevés de blocs (le dessin des formes complexes est facilité).

- soit pour du stockage d'information archéologique pour un traitement différé ou pour garder le maximum d'informations sur des zones qui ne pourront plus être fouillées.

- soit comme substitut aux moulages traditionnels en plâtre. Il devient assez simple de mémoriser un foyer magdalénien, par exemple, (selon les cas, une centaine d'images peuvent suffire) et de pouvoir revenir prendre des mesures ou visualiser en situation la couche archéologique démontée. Par le biais du prototypage, il est possible de produire un positif en matériaux synthétiques pour une éventuelle valorisation.

De nombreuses applications sont donc possibles dans le domaine de l'archéologie du bâti, de l'épigraphie, de la Préhistoire, etc. que ce soit en contexte de fouilles programmées ou de sauvegardes. La numérisation ne se limite pas aux sites, des objets peuvent aussi être numérisés, là encore les applications sont nombreuses, études physiques, conservation de l'information $3 \mathrm{D}$, valorisation, etc. [ill. 4].

\footnotetext{
Références bibliographiques

Furukawa Y., PONCE J., 2009, "Accurate, Dense, and Robust Multi-View Stereopsis", IEEE Trans, on Pattern Analysis and Machine Intelligence.

Lowe D. G., 2004, "Distinctive image features from scale-invariant keypoints", International Journal of Computer Vision, 6o, 2, pp. 91-110.

Pierrot-Deseilligny M., Paparoditis N., 2006, "A multiresolution and optimization-based image matching approach: An application to surface reconstruction from $\mathrm{SPOT}_{5}$-HRS stereo imagery", in IAPRS vol XXXVI-1/W41, ISPRS Workshop On Topographic Mapping From Space (With Special Emphasis on Small Satellites), Ankara, Turquie.

Snavely N., Seitz S. M., Szeliski R., 2007, "Modeling the World from Internet Photo Collections", International Journal of Computer Vision.

Snavely N., Seitz S. M., Szeliski R., 2006, "Photo Tourism: Exploring image collections in $3 \mathrm{D}$ ", $A C M$ Transactions on Graphics (Proceedings of SIGGRAPH 2006),

Vergne R., PaCANowski R., Barla P., Granier X. SCHLICK C., 2010, Radiance Scaling for Versatile Surface Enhancement, 3 D'10, Proc. Symposium on Interactive ${ }_{3} \mathrm{D}$ graphics and games.
} 


\section{1}

unité $=\mathrm{cm}$

$=0$

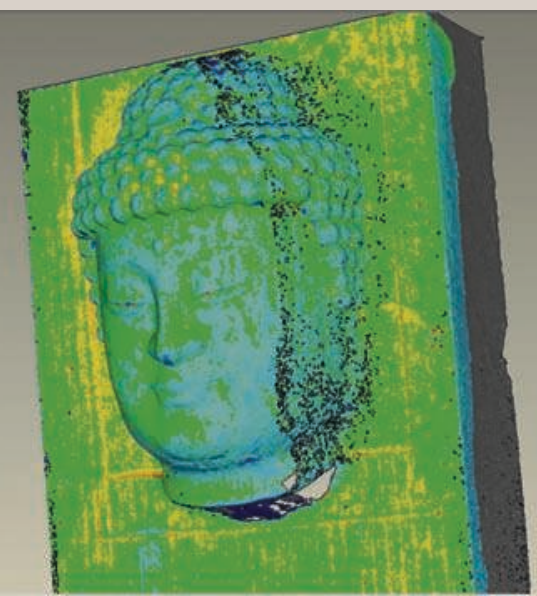

photogrammétrie par

corrélation d'images sur

des chantiers de taille très

différente. Une monnaie

romaine $(2 \mathrm{~cm})$, un ensemble

monumental (zone d'environ

1 ha) traité en ortho-image

pour la DAO du relevé pierre

à pierre. Les points et tracés

rouges sont des relevés faits

au tachéomètre. Ces mesures

sont utiles sur les gros

chantiers car elles permettent une mise à l'échelle et une orientation rapide des modèles 3D. De plus, il est plus

pratique de traiter les images en plusieurs fois, ceci évite

les calculs trop longs.

4. De gauche à droite, nuage

de points bruts, modèle maillé

et texturé, visualisation

à l'aide d'un rendu en radiance

scaling (R. Vergne et al.

2010), les deux dernières

images sont en mode

d'affichage orthographique.

3. Quelques exemples

d'application de la

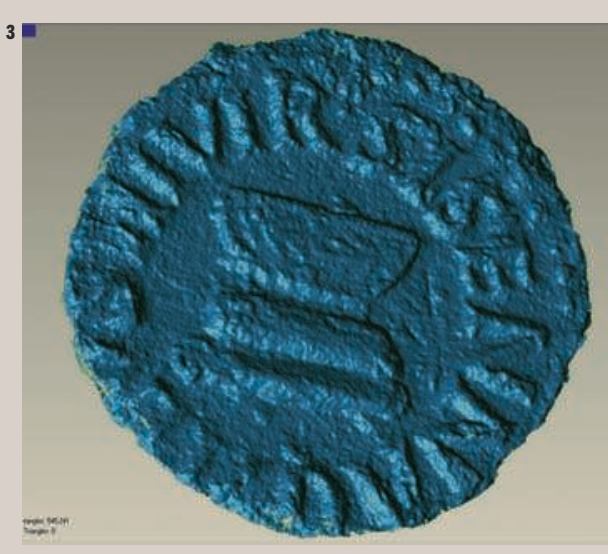

$\Rightarrow$

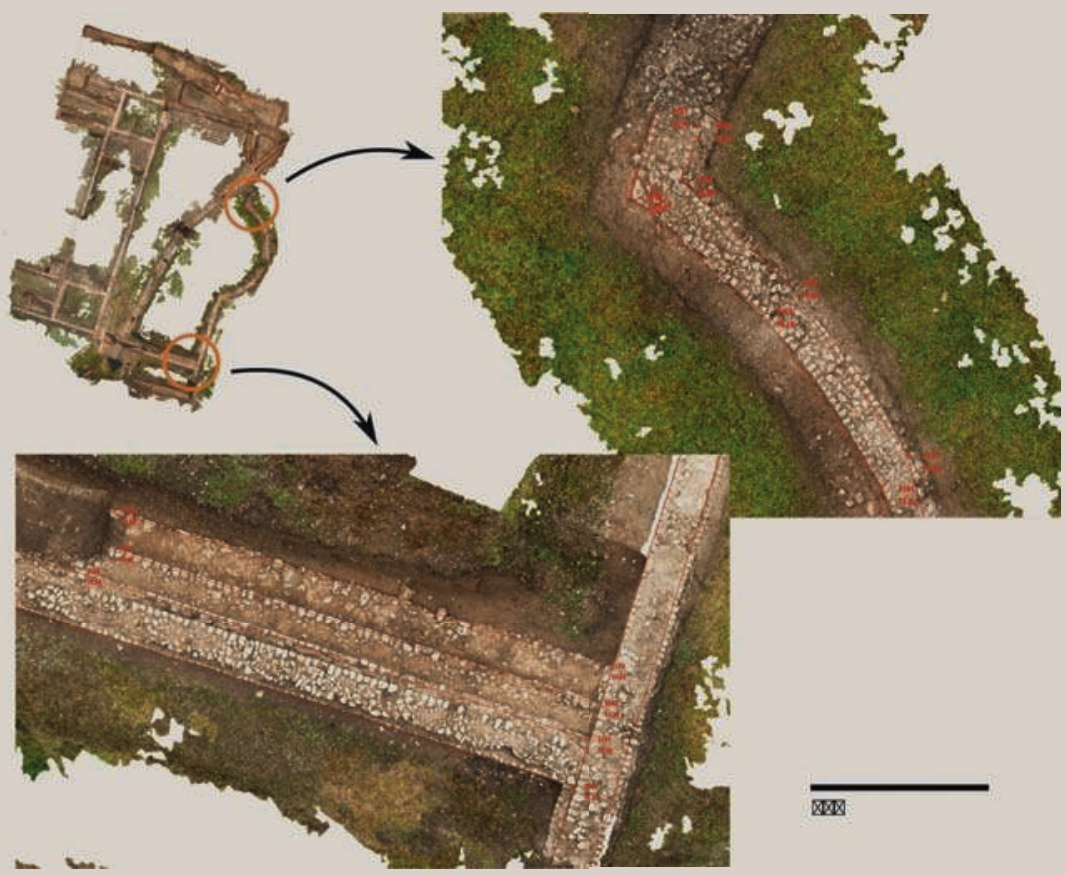

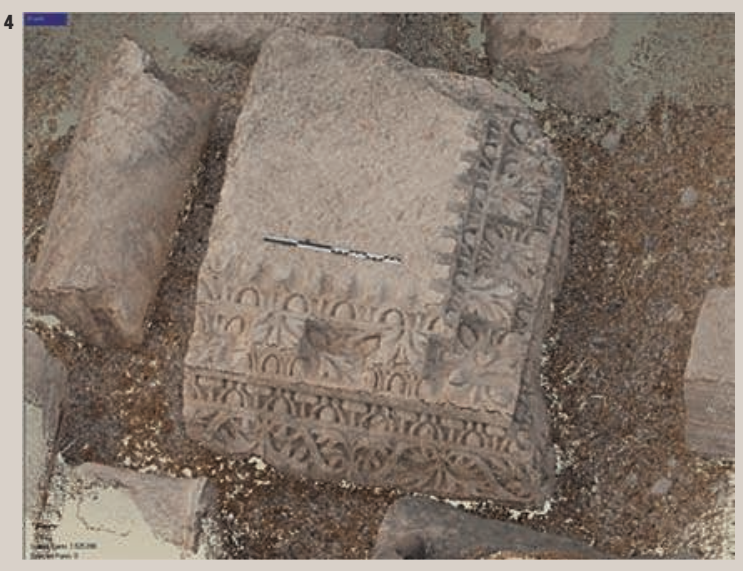
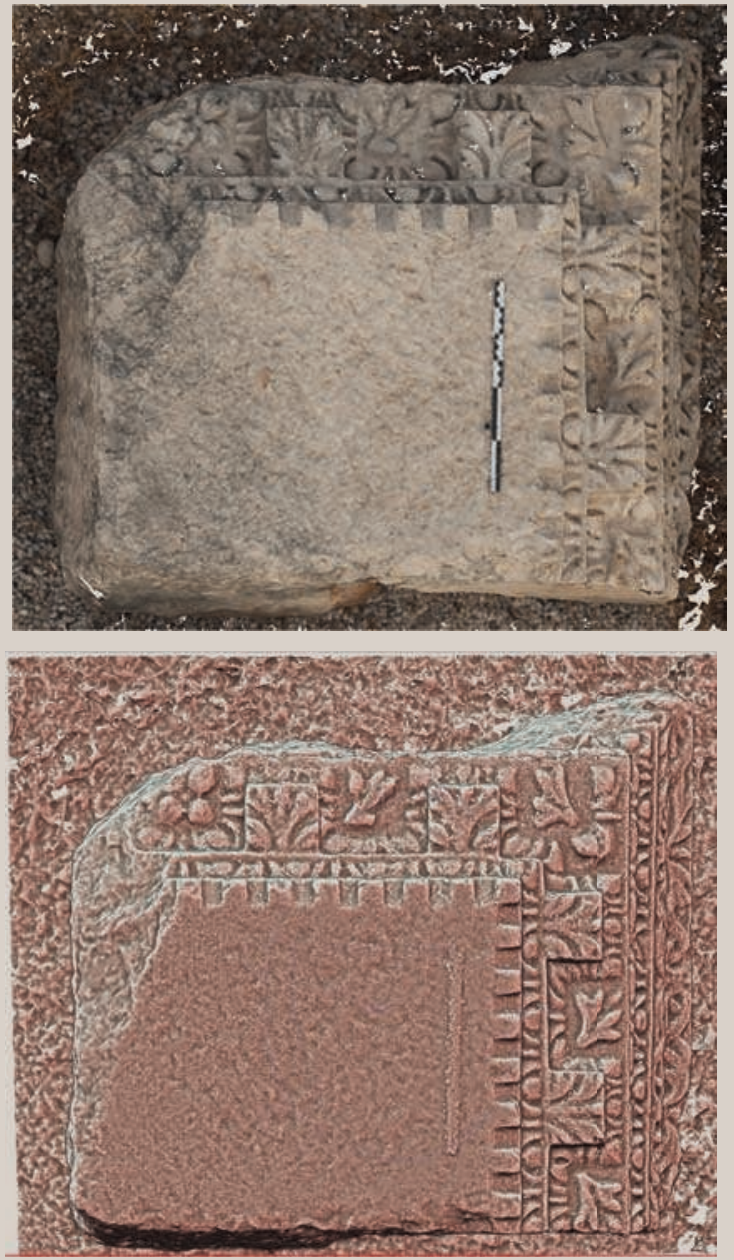